\title{
Représentations de la filiation dans les littératures francophone et autochtone contemporaines du Canada dans le contexte des
}

\author{
Amériques.
}

Representations of parentage in contemporary Francophone and Aboriginal literature in Canada in the context of the Americas.

\author{
Adina Balint ${ }^{1}$ \\ Patrick Imbert ${ }^{2} 3$
}

Submetido em 24 de novembro e aprovado em 2 de dezembro de 2019.

Résumé: Cet article dont la réflexion se situe à la croisée des études littéraires, des études comparatistes et transculturelles explore différentes représentations de la filiation (familiale, culturelle et communautaire) dans les littératures francophone et autochtone contemporaines du Canada, dans le contexte des Amériques. On rapproche ainsi des textes d'un écrivain québécois comme Éric Dupont, un récit autobiographique d'une écrivaine canadienne anglophone, Jenny Heijun Wills, et un témoignage d'une auteure autochtone, Jane Willis, par la mise en tension entre le sujet littéraire, la lignée familiale et culturelle et la communauté. Ayant recours aux notions de «bâtard» et «d'orphelin» de Marthe Robert et au concept de «nomadisme» de Rosi Braidotti, nous réfléchissons à de nouvelles figures d'appartenance, au-delà de l'exclusion. Nous montrons que la filiation n'est pas une donnée fixe et immuable mais un processus inachevé/inachevable, catalyseur de créativité.

Mots-clés: Fikiation. Littérature francophone contemporaine du Canada. Littérature autochtone du Canada. Éric Dupont. Jane Willis. Jenny Heijun Wills. Orphelin. Bâtard.

Abstract: At the intersection of literary, comparative and transcultural studies, this article explores various representations of filiation (among the family, culturally and via the community) in contemporary Francophone and Indigenous literatures of Canada as part of the Americas. This approach leads us to compare texts by the Quebecois writer Éric Dupont, a memoir by the Anglophone-Canadian Jenny Heijun Wills and a testimonial by the Indigenous author Jane Willis. We focus on the tensions between the literary subject, the family genealogy, the cultural background and the community. Through the theoretical notions of "bastard" and "orphan" in Marthe Robert's work and through the conception of "nomadism" in Rosi Braidotti, we study new figures of belonging beyond exclusion. Thus, we show that filiation is not only fixed data but an ongoing process driven by and driving creativity.

Keywords: Parentage. Contemporary Francophone Literature of Canada. Indigenous Literature of Canada. Éric Dupont. Jane Willis. Jenny Heijun Wills. Orphan. Bastard. 


\section{Introduction}

Marthe Robert (1972) affirme que, dans les Amériques, pour les descendants des settlers, pour les Créoles, l'identité du Nouveau Monde tend à osciller entre l'orphelin et le bâtard. L'orphelin est celui qui cherche constamment ses parents, qui est rongé par la nostalgie et qui vise à être généré par une reconnaissance, par des ancêtres généralement européens. Le bâtard est celui qui veut recommencer à neuf, se créer, choisir ses parents, inventer ses ancêtres, devenir un self-making man ou une self-making woman et s'affirmer en fonction d'une légitimité des mobilités et des déplacements géo-symboliques.

\section{La filiation}

Mais comment assure-t-on une filiation? Par la transmission de textes canoniques juridiques, religieux, scientifiques, littéraires et artistiques. Comme le souligne Lelia Area (2006) dans Una biblioteca para leer la Nación, les Amériques issues du mythe du progrès positiviste et dualiste du dix-neuvième siècle sont inventées par les livres européens dont la civilisation est conçue comme supérieure. Le texte européen reposant sur le paradigme barbarie/civilisation produit le texte des Amériques et invente le discours sur l'espace et sur celles et ceux qui l'habitent. Celui-ci situe le settler, le Créole entre deux altérités. D'une part, on a l'être européen «supérieur» auquel on a accès dans les ports des Amériques tournés vers l'Europe, et d'autre part, l'Autochtone ou le Noir «inférieur», sans littérature, donc barbare, souvent lié à l'arrière-pays ou en fuite, et fondant des Quilombos, des zones indépendantes. Il y a danger de barbarie aussi pour certains descendants de l'Europe, par exemple les Canadiens français dont Lord Durham affirme qu'ils «sont un peuple sans histoire et sans littérature» (BERTRAND ET LAVALLÉE, dir., 1969, p. 17). Les enfants qui s'affirment du sol doivent donc produire une littérature pour être reconnus comme Nation. La littérature et son absence servent à exclure car la civilisation, c'est se rassembler autour d'élites qui ont pour rôle d'inventer une Nation homogène par le biais d'une littérature et d'une langue partagées. Cela implique, comme le rappelle Shapiro (1997), d'exclure certains groupes considérés comme inassimilables, car ils sont perçus comme non-rationnels, comme les Noirs et les Autochtones, ainsi que de minoriser les femmes. Sarmiento l'exprime bien dans Facundo en 1845 par la diffusion 
de son paradigme barbarie/civilisation repris des textes scientifiques issus de l'Europe. Ce paradigme dualiste barbarie/civilisation, synonyme de soi/les autres, est présenté en fonction d'une base scientifique indiscutable au dix-neuvième siècle, notamment par Alfred P. Schultz dans Race of Mongrel ${ }^{4}$ en 1908 et par le professeur de Harvard Louis Agassiz dans Journey in Brazil où l'on peut lire ce qui suit:

The natural result of an uninterrupted contact of half-breeds with one another is a class of men in which pure type fades away as completely as do all the good qualities, physical and moral, of the primitive races, engendering a mongrel crowd as repulsive as the mongrel dogs, which are apt to be their companions, and among which it is impossible to pick out a single specimen retaining intelligence, the nobility, or the affectionateness of nature which makes the dog of pure type the favorite companion of civilized man. (p. 26)

Voilà qui est clair et d'un racisme scientifique ${ }^{6}$ imbu de toute sa certitude progressiste. Les volumes de Schultz et d'Agassiz ont été très populaires et ont connu de nombreuses rééditions. Tous deux contribuent à démontrer l'infériorité barbare de tous ceux qui sont issus de mélanges. Autrement dit, la filiation doit reposer sur la pureté de la race.

Mais qu'en est-il de nos jours du penchant vers l'orphelin ? Pour l'évaluer on va se consacrer à deux textes récents: Older Sister. Not Necessarily Related (2019) de l'écrivaine canadienne anglophone d'origine coréenne Jenny Heijun Wills et La Route du lilas (2018) de l'écrivain québécois Éric Dupont. Dans le premier cas, il s'agit d'un bébé fille abandonné par ses parents en Corée du Sud et adopté à l'âge de neuf mois par une famille anglo-canadienne de l'Ontario. Il s'agit d'un texte autobiographique (le mot «a memoir» apparaît sur la couverture): la narratrice anonyme du livre a des liens biographiques avec l'auteure, elle-même d'origine coréenne et adoptée par une famille ontarienne et qui tisse une réflexion sur l'exclusion et des formes sous-jacentes de colonialisme. Dans le deuxième cas, celui d'Éric Dupont, il s'agit d'un récit complexe de plus de 560 pages à travers le continent américain avec un passage par l'Europe, le tout ouvrant sur une recherche aventureuse par les enfants pour savoir, qui était la «résilienne Pia», la mère de Simone et qui était la Québécoise Thérèse, la mère de Rosa Ost, déjà présente dans le roman La Logeuse d'Éric Dupont (2006). 


\section{La tendance vers le bâtard}

Souvent, le côté bâtard n'est pas vécu dans l'euphorie sauf aux États-Unis qui font la révolution, rejettent la colonisation par l'Angleterre et valorisent le self-made man pour affirmer leur pouvoir. Simultanément, les États-Unis détruisent les cultures autochtones et perpétuent jusque dans les années 1960 une féroce discrimination envers les Afro-descendants. Au Québec, Gérard Bouchard parle de la stratégie du bâtard qui est une forme d'anthropophagie qui n'est ni expansive ni conquérante mais tournée contre soi-même: «the founding culture sustains itself not on the wealth of others, but rather, on its own poverty, on its real or imaginary lacks, through quiet and voracious subversion» (2008, p. 314) ${ }^{7}$, ce qui pourrait être le cas pour Bérénice Einberg dans L'Avalée des avalés de Réjean Ducharme. Autrement dit, il manque l'accès aux autres, la capacité de valoriser la rencontre, tout ce qu'on trouve chez Oswald de Andrade, notamment dans le Manifeste anthropophage pour produire une dynamique qui nourrit et renouvelle les rapports sociaux. Bouchard souligne, de plus, que le journaliste Richard Gwynn affirme que: «Canadians should consider themselves a nation of 'mutts', entirely created, autonomous from birth» (p. 313) ${ }^{8}$. Il va à l'encontre d'une tradition de penseurs qui veulent fonder des nations qui soient fortes et légitimes et pour cela, il faut que les identités ne soient pas créées de toutes pièces mais générées. Il faut que les identités soient branchées sur un lignage, sur une filiation, sur une continuité longue. Le désir d'autogénération qui tient sa force de l'Europe ne peut fonctionner dans les Amériques qui n'ont pas de base historique, culturelle et architecturale longue sauf bien sûr pour les Autochtones mais, dans l'optique coloniale, ils sont des barbares et ne peuvent être considérés comme des moteurs d'un lignage. Le métissage, les Métis sont exclus, comme on le voit pour Louis Riel. Gwynn s'oriente ainsi vers une vision inédite des identités au Canada, vers une perspective positive sur le bâtard, celui qui invente ses parents et se tourne vers un avenir construit par la rencontre des altérités. Une vision nouvelle de la nation se thématise, ce qui rejoint la conclusion de Pico Iyer dans Imagining Canada:

I end now, at long last, with a confession : throughout this talk I've used the highly unlovely word 'mongrel' instead of the euphonious government-approved word, 'hybrid' [...] As Michael 
Ondaatje suggests with the use of the equally unsentimental term, 'international Bastards', the process of mixing may not be pretty, but it's no bad thing if one comes without a pedigree. (p. 56)

On voit que Marthe Robert et sa théorie de la création littéraire issue du fait que l'enfant se raconte la fiction qu'il est soit orphelin, soit bâtard, entretient un lien particulier avec les Amériques même si elle ne considère pas les Autochtones (qui sont, ni orphelins ni bâtards) puisque dans le discours colonial ils sont barbares. Marthe Robert souligne que l'enfant bâtard, différemment de l'EEdipe, «ne tue pas son père, il le supprime tout simplement du cercle familial, mais en s'octroyant le père idéal dont il espère s'approprier les qualités» (p. 53): intelligence, richesse, indépendance, ce qui est une des bases de la dynamique menant au self-made man (MAXWELL, 2009).

Dans le monde contemporain jouant des mobilités sociales, éducatives, technologiques et économiques, le père idéal, le modèle dont il faut prendre l'objet désirable selon la perspective de René Girard (1978), est représenté par des gens ouvrant sur les relations planétaires et l'accès aux savoirs, Bill Gates, Mark Zuckerberg, Steve Jobs. Le modèle n'est pas donné ni établi dans le long terme; il est construit selon les besoins du moment en un acte de langage qui fait que l'on se promet le père que l'on souhaite avoir. Ainsi, on s'engage dans une très singulière déclaration d'indépendance où l'on s'appartient plus qu'on appartient à un groupe et à ses ancêtres dont on est orphelin ou pas. On vise alors, dans l'ambition la plus grande, des changements qui permettront d'étendre les capacités et l'accès aux réseaux. C'est ce qu'on lit chez Amy Tan:

\footnotetext{
My mother believed you could be anything you wanted to be in America...America was where all my mother's hopes lay. She had come here in 1949 after losing everything in China: her mother and father, her family, home, her first husband, and two daughters, twin baby girls. But she never looked back with regret. There were so many ways for things to get better. (p. 56) ${ }^{10}$
}

On se dirige nettement vers la démarche non pas du self-made man mais du selfmaking man et de la self-making woman. Voilà qui évoque aussi la phrase d'un personnage de Dany Laferrière dans Cette grenade dans la main du jeune nègre est-elle une arme ou 
un fruit. Il y affirme qu'il veut l'Amérique toute entière et qu'il l'aura. On pense aussi à tous les livres de Kim Thúy. Il est d'ailleurs intéressant de constater que beaucoup d'écrivains qui ne sont pas nés dans les Amériques présentent leurs personnages avec des rêves similaires, donc plus comme des bâtards inventant leurs ancêtres que des orphelins nostalgiques, ce qui devrait nourrir la réflexion des théoriciens du multiculturalisme comme Will Kymlicka (2007) ou Charles Taylor (1994).

\section{Jenny Heijun Willis: une narratrice orpheline?}

Le livre de Jenny Heijun Willis Older Sister, Not Necessarily Related est une réflexion psycho-poétique et fragmentée d'une narratrice adoptée par des parents anglo-canadiens. Elle vit diverses formes d'exclusion, notamment à l'école ce qui lui fait bien saisir qu'elle n'est pas conforme au désir de la communauté locale blanche. On lui demande si elle parle chinois, on se moque d'elle, etc. (2019, p. 32, 67, 89). La situation est d'autant plus grave qu'elle n'est pas une immigrante. Une immigrante qui subit racisme et exclusion au Canada peut se référer à la culture, à la langue et parfois à la communauté d'où elle est issue pour continuer à garder son équilibre symbolique et narratif. Ce n'est pas le cas de la narratrice de Willis qui est arrivée au Canada à neuf mois et qui ne connaît pas d'autre langue que l'anglais (et apprend le français) ni d'autre culture que celle de l'Ontario. Le problème est alors immense car elle n'a pas d'autre choix que de s'inventer dans une culture qui ne veut pas d'elle et qu'elle doit intégrer malgré tout. Il n'y a pas d'autre chemin que le retour aux couches, à la mère qui l'a abandonnée, à la Corée du Sud qui pourra combler sa sensation d'être intensément orpheline. Ainsi, elle pourra s'inventer comme immigrante, comme biculturelle puisque la mono culture anglo-canadienne lui impose le racisme. La filiation se trouve ailleurs et notamment dans la capacité à symboliser le malaise par l'écriture. Dès lors, en plus de construire un capital génétique et culturel, il est possible de se recréer dans le symbolique, c'est-à-dire de s'inventer une filiation artistique par l'intermédiaire d'écrivaines qui inspirent. Dans des entretiens récents (KOEVERDEN, 2019), Jenny Heijun Wills affirme se nourrir de littérature ethnique, par exemple. 


\section{Éric Dupont: les personnages féminins à la fois orphelins et bâtards?}

La caricature des allégeances et des appartenances est un des motifs des romans d'Éric Dupont. C'était le cas dans La Logeuse où Rosa Ost a fui l'univers national et marxiste de la petite communauté dictatoriale de Notre-Dame-du-Cachalot en Gaspésie. Dans La Route du lilas, on se consacre à explorer les déplacements géo-symboliques plutôt ludiques et positifs. Parmi tous les fils narratifs sur lesquels plane le vol des engoulevents, oiseaux migrateurs allant de l'Amérique du Sud à l'Amérique du Nord comme l'évoque aussi Jacques Ferron dans La nuit, il s'agit de Maria Pia, une Brésilienne qui se rend à Paris avec son mari Thiago, plutôt violent. Elle y rencontre la Québécoise Thérèse qui méprise sa famille du Canada. Les deux femmes tombent amoureuses. Par la suite, Maria Pia accouche de Simone. Thérèse la laisse tomber en précisant qu'elle part, car elle voit que Maria Pia a peur d'une vraie liberté. Déjà là, on sent les pôles se préciser. Thérèse est plutôt pour s'inventer des ancêtres et une vie hors des normes. Pia est plus tenue dans la tradition et ce n'est que plus tard qu'elle quittera le Brésil et fera la route du lilas aux États-Unis et au Canada avec Shelly et Laura, jusqu'à Montréal où elle retrouvera la fille de Thérèse, Rosa Ost. La ressemblance entre Thérèse et Rosa est si forte que Pia décide de vivre avec elle: «Elle (Simone) a pour ainsi dire disparu de ma vie. Je n’ai plus de fille. Et toi, tu n'as plus de mère» (La Route du lilas, p. 520). Pia prend pour fille adoptive Rosa car sa fille Simone l'a rejetée alors qu'elle recherche son père probablement éliminé par la dictature: «Et le cinéma mental des orphelins recommence à tourner dans sa tête, et repartent ces jeux morbides comme celui qui consiste, dans un restaurant, à se dire: 'le prochain qui entre, c'est mon père'» (p. 401). Les Amériques se cherchent dans une cacophonie des lignages et des descendances à rejeter, à rechercher, à inventer: «Les orphelins comprennent avant tout le monde que les parents ne sont finalement qu'un récit, une histoire, un conte pour dormir [...]» (p. 403). Toutefois, dans ce roman, les orphelins ont décidé de ne plus être orphelins ce qui rejoint des problématiques plus nationales: «Nous étions du Nouveau Monde, trouvions les Français un peu figés dans leurs manières, parlions avec un accent, bref, nous étions les Autres» (p. 163). Autrement dit, les gens des Amériques ne reconnaissent plus les canons européens et sont capables de s'inventer dans leurs multiplicités sans passer par l'Europe, comme le propose Zilá Bernd (2015): il 
est possible de se recréer dans le symbolique. Déclaration d'indépendance et de créativité fictionnelle, La Route du lilas voyage sur les possibles offerts par des choix de s'inscrire comme on le veut dans des vies renouvelées tout en sachant «comment le monde s'était organisé avant sa naissance» (p. 455). Quant à Simone invitée par Pia «à marcher dans un récit plus grand que sa propre personne» (p. 589), elle est «la somme de toutes ces femmes» (p. 589).

Par récit plus grand que sa propre personne, on entend la capacité à dépasser une autobiographie centrée sur l'individu pour sonder la grande chaîne des êtres reliés génétiquement, symboliquement et scripturairement par un intertexte qui fait se recouper La Logeuse et La Route du lilas en fonction de personnages, comme Rosa Ost, par exemple, qui reparaissent comme dans La Comédie humaine de Balzac. La filiation est aussi une filiation narrative qui invente des déplacements culturels et sociétaux où les autres ont leur place et où les orientations sexuelles comme les amours se choisissent du Brésil au Canada.

\section{Les Autochtones, ni orphelins, ni bâtards.}

La dynamique explorée par Marthe Robert, l'oscillation entre orphelin et bâtard dans un Nouveau monde en gestation ne concerne pas les Autochtones. Ils sont liés à une durée longue, à des cultures et à un territoire, ce qu'on leur a nié jusqu'à récemment. On retient par exemple les pensionnats où on enfermait les enfants autochtones arrachés de force à leurs parents pour retirer l'Indien en eux et en faire des personnes similaires aux Blancs, une dynamique qui s'apparente au résultat potentiel de colonisé acceptable, donc de not quite au sens de Homi Bhabha (1993). Leur présence et leur culture ont donc été exclues et cette exclusion s'est perpétuée jusqu'à récemment. Quant à la terreur liée à l'enfermement et aux violences dans les pensionnats, peu de Canadiens non-autochtones savaient ce qu'il se passait même si quelques informations ont circulé dans des magazines à grande diffusion, comme dans le magazine MacLeans en février 1967. Ce magazine a publié l'histoire de Chanie Wenjack qui avait 12 ans quand il a fui la violence de l'école résidentielle à Kenora (Nord de l'Ontario), et qu'il est décédé quelques jours plus tard (ADAMS, 1967, p. 37-45). L'article a été vite oublié. 
Une absence d'écoute s'est manifestée aussi pour le livre de Jane Willis intitulé Geniesh: An Indian Girlhood ${ }^{11}$. Le livre a été publié en 1973. Jane Willis y relate son expérience très négative dans un pensionnat au Québec et le décès d'une pensionnaire qui n'a pas été soignée. Linda Warley qui a publié un article en 1998 au sujet de ce livre souligne qu'il n'y a eu que deux comptes rendus à l'époque, l'un dans Chatelaine magazine en 1973 (CHARNEY, p. 12) et l'autre dans Canadian Forum (CHEDA, p. 41-42) en 1974. Dans ce dernier, Sherill Cheda souligne bien les conditions horribles infligées aux enfants Autochtones qui sont celles des prisons. Par contre, les commentaires de Penny Petrone (1990), dans son livre sur la littérature des Autochtones au Canada, rejoignent une tradition de la critique littéraire qui rejette les écrits autochtones comme ne faisant pas partie de la littérature canadienne: «Willis's account of her early life and education does not rise above autobiographical protest because she has not moved beyond bitterness and the desire to condemn» $(\text { p. 117 })^{12}$. Voilà qui manque singulièrement de compréhension et qui n'aide guère à la diffusion du livre de Jane Willis. On pense aussi à Je suis une maudite sauvagesse de An Antane Kapesh publié en 1976, mais qui n'a guère été diffusé et qui n'a été republié qu'en 2019 par la maison d'édition Mémoire d'encrier à Montréal.

Autrement dit, la situation politique colonialiste refuse toute communication et toute relation de métissage entre les cultures. Il faut tout simplement assimiler les Autochtones en brisant leurs filiations traditionnelles et en rejetant les mélanges possibles. C'est bien pour cela que Naomi Fontaine reconnaît que pendant un certain temps, elle a «créé un monde faux» (Kuessipan, p. 9), celui où «les mères font des enfants pour les aimer, où on fait survivre la langue» (Kuessipan, p. 9). Depuis, cette auteure raconte autre chose : un monde d'exclusion, de désolation parfois, mais qui est en train de se régénérer, un monde qui se reconstruit économiquement et symboliquement.

Voilà aussi une position qui est explorée par l'artiste peintre canadien des Premières Nations, d'origine crie et irlandaise, Kent Monkman, dont les tableauxfresques d'une facture réaliste alternative pastichant les codes européens - donc bien éloignés des tableaux inspirés par les cultures autochtones traditionnelles comme ceux de Jackson Beardy ou de Benjamin Chee Chee - présentent les violences de la colonisation tout en générant une hybridité qui demande reconnaissance ${ }^{13}$. Kent Monkman souligne, 
par exemple, dans le livret intitulé Miss Chief's Memoirs, accompagnant l'exposition itinérante intitulée Shame and Prejudice: A Story of Resilience, que l'appropriation des éléments esthétiques et symboliques autochtones par le Cubisme de Picasso renouvelait l'art européen au moment même où les Autochtones étaient enfermés dans des réserves et des pensionnats-prisons tandis que leurs langues et cultures étaient détruites (p. 9). Un de ses tableaux dépeint d'ailleurs les prêtres et les agents de la police montée en train de kidnapper les enfants dans un village autochtone. Un autre des tableaux de Monkman, intitulé The Daddies, produit en 2016, parodie violemment la respectabilité des institutions historiques canadiennes ${ }^{14}$. On y voit les figures clés liées à la Confédération canadienne et, face aux pères de la Confédération, Miss Chief nu/e, un personnage au double esprit (two spirit), queer, et assis/e sur une couverture de la compagnie de la Baie d'Hudson. Elle/il impose la présence autochtone dans les négociations concernant cette Confédération canadienne.

Ainsi, le but de Kent Monkman va plus loin que de diffuser les souffrances des peuples autochtones et de demander de reconnaître le génocide qui s'étale sur 150 ans depuis la Confédération de 1867: «My mission is to authorize Indigenous experience in the canon of art history that has heretofore erased us from view» $(2017, \text { p. } 4)^{15}$. Il s'agit de réécrire l'histoire du Canada, de produire des manuels pédagogiques qui reconnaissent les violences faites aux Autochtones comme leur apport à la société canadienne avant la Confédération et depuis celle-ci. Il s'agit de produire une nouvelle narrativité institutionnelle et canonique, de pénétrer et de transformer le centre du pouvoir symbolique pour donner une place juste à toutes celles et à tous ceux et qui ont contribué et contribuent à construire le Canada et cela afin que les enfants à l'école comprennent autrement et mieux leur pays et celui de leurs ancêtres. La filiation dominante des Blancs est déjouée par un métissage symbolique et narratif qui transforme les deux cultures.

C'est pour cela que le livre intitulé As We Have Always Done (2017) de la Canadienne autochtone Leanne Betasamosake Simpson est particulièrement intéressant. Il ouvre directement sur les paradigmes sur lesquels reposent la plupart des langues autochtones et sur une narrativité contemporaine et militante qui raconte l'histoire de ce qu'ont vécu les peuples autochtones. Betasamosake Simpson insiste surtout sur un élément 
extrêmement important qui déplace la notion même de norme et d'hétéronormativité transmise de père en fils, dans un mouvement de nomadisme symbolique: «It led me to consider what straightness looks like in societies where queerness is normalized, where difference isn't difference but normal» (p. 129) ${ }^{16}$.

\section{Le nomadisme comme posture esthétique}

Dans son ouvrage Nomadic Subjects, la théorecienne Rosi Braidotti examine la question du nomadisme en rapport avec la subjectivité. À la recherche d'un savoir féministe non normatif, Braidotti en vient à proposer un féminisme critique et créatif, fondé sur le nomadisme. Selon elle, le sujet nomade incarne une «fiction politique», étant capable de brouiller les frontièrex: «The nomadic subject is a myth, that is to say a political fiction, that allows me to think through and move across established categories and levels of experience: blurring boundaries without burning bridges» $(2011, \text { p. } 4)^{17}$. Si Braidotti retient la figure mythique et iconoclaste du nomade, c'est qu'elle occasionne une démarche en opposition à la nature sédentaire et classique de la pensée théorique et surtout, philosophique, en permettant l'ouverture à de nouvelles complicités et formes d'interaction au-delà de la partialité et de l'intermittence :

The choice of an iconoclastic, mythic figure such as the nomadic subject is consequently a move against the settled and conventional nature of theoretical and especially philosophical thinking. This figuration translates therefore my desire to explore and legitimate political agency, while taking as historical evidence the decline of metaphysically fixed, steady identities. One of the issues at stake here is how to reconcile partiality and discontinuity with the construction of new forms of interrelatedness and collective political projects. (p. $4-5)^{18}$

En outre, la posture nomade désigne ici la subversion des conventions et non l'acte physique de voyager: «The nomad does not stand for homelessness, or compulsive displacement; it is rather a figuration for the kind of subject who has relinquished all idea, desire, and nostalgia for fixity» (p. 22) ${ }^{19}$. L'essence même du sujet nomade est d'être «postidentitaire»: «nomade est un verbe, un processus à travers lequel nous dressons la carte des transformations multiples et des multiples modes d'appartenance [...]» (BRAIDOTTI, «Sur le nomadisme : entretiens avec Rosi Braidotti»). Ou encore: “The nomadic subject 
[...] is not devoid of unity; his/her mode is one of definite, seasonal patterns of movement through rather fixed routes. It is a cohesion engendered by repetitions, cyclical moves, rhythmical displacements" (BRAIDOTTI, 2011, p. 22) ${ }^{20}$. Une cohésion apparaît au sein des répétitions du sujet nomade et de ses mouvements cycliques inspirés par les saisons. De plus, contrairement à l'agriculteur, le nomade rassemble, récolte, échange, mais n'exploite pas. Cette pratique respectueuse de l'environnement rejoint les modes de vie et de pensée des peuples autochtones du Canada, tel que le montre Leanne Betasamosake Simpson dans son ouvrage As We Have Always Done, où elle revendique une «land-based pedagogy» («pédagogie de l'habitation de la terre») ${ }^{21}$ en tant que processus susceptible de mener à une revalorisation des savoirs et des valeurs Nishnaabeg, au préalable nourris par la terre.

$\mathrm{Au}$ fond, la posture existentielle et intellectuelle du nomade ou de la nomade ne consiste pas à prendre les traits du sans-abri, mais à recréer un chez-soi partout sans que celui-ci prenne racine: «As an intellectual style, nomadism consists not so much in being homeless, as in being capable of recreating your home everywhere. The nomad carries her/his essential belongings with her/his wherever s/he goes and can recreate a home base anywhere» (2011, p. 16) 22, rappelle Rosi Braidotti. À cet effet, tout comme Deleuze et Guattari, Braidotti distingue le nomade de l'exilé et du migrant qui désirent se reterritorialiser. La mobilité du nomade n'est pas de l'ordre de l'itinérance ni des déplacements compulsifs. Elle incarne plutôt la traversée des frontières ou plutôt la circulation sans frontières, l'acte de cheminer, l'art d'être en mouvement indépendamment de la destination. En cela, force est de constater que les littératures québécoise et francophones du Canada, de même que la littérature française de l'extrême contemporain, n'échappent pas à cette recherche d'actualisation culturelle et identitaire qui traverserait les frontières pour permettre de s'ancrer dans des textes hybrides ou nomades, comme Cybermigrances de Régine Robin, par exemple, ou encore, comme L'Énigme du retour de Dany Laferrière. Au cœur des «lieux» que les écrivains représentent, on retrouve en effet des espaces hétérogènes qui dépassent la fixité référentielle et suggèrent une interrogation renouvelée des figures du «nomadisme». En déplaçant les lieux référentiels vers des espaces de l'imaginaire et du fantasme, les écrivains sémantisent une relation 
au territoire qui se pense à la jonction des représentations géographiques et de l'espace poétique, tout en questionnant le sentiment d'appartenance à un territoire (qui serait celui de l'écriture) et à une identité multiple. À la fin de Cybermigrance, dans un bref chapitre intitulé « Fourre-tout », la narratrice errante avoue :

Je rêve d'un journal qui me rassemblerait. Un fourre-tout mais dans lequel on se repérerait malgré tout, qui consignerait à la fois les rêves, les rêvasseries, les fantasmes, les projets, les réflexions, les citations, les remarques de lecture. Le tout comme un collage sans ordre, mais on ne s'y perdrait pas pour autant; pas tout à fait. [...] On prendrait des lignes de métro avec de multiples correspondances qui ne correspondraient à rien. Ni archéologie, ni hiéroglyphes, la vie comme une déambulation urbaine. (p. 237)

Sans perdre de vue les assises épistémologiques d'un mode de pensée nomade, cette définition de l'écriture comme nomadisme ou du nomadisme comme écriture, permet de réfléchir à l'expérience de la mobilité selon des filiations symboliques et métaphoriques, en plus d'interroger les relations entre le sujet narrateur ou narratrice et son désir de se reterritorialiser. L'entrecroisement de l'univers référentiel et poétique (imaginaire, fantasmatique) est aussi l'occasion de mettre à jour les malaises et les ambivalences provoquées par la création de ces espaces textuels dans les récits qui sont bel et bien le reflet de sujets nomades en quête de nouvelles lignes de créativité. À ce propos, il faut savoir que le désir d'ancrage - ne serait-il qu'illusoire, mais propre à l'être humain oscille entre un principe de stabilité, qui emprunte à l'imaginaire de la terre natale, et une ambition de se démarquer. Cette dernière se définit par l'explosion des frontières et par la mise en crise de l'expérience de la sédentarité à travers la migration.

\section{Conclusion}

Qu'elle se marque par l'échec ou qu'elle atteigne son but, la mobilité physique, symbolique et des imaginaires traverse et structure l'exploration de la filiation conduite chez les auteur.es étudié.es. En posture d'héritiers entre mères, pères, ascendants perdus ou oubliés, ainsi qu'en posture de dissidents face à des héritages coloniaux, les personnages et narrateurs/narratrices n'hésitent pas à effectuer une retraversée des lieux communs afin d'arriver à de nouvelles modalités de la filiation, placées sous le signe du 
questionnement et de la quête comme politique créatrice de liens. À partir des ruines, des restes, de l'ancien et d'un nouveau à réinventer, les récits travaillent à créer du lien dans et par la dislocation, le mouvement et la mise à distance. Évidemment, le questionnement du lien de filiation ne se limite pas, chez les auteur.es exploré.es, au contexte familial ; parmi les autres types de filiation et d'affiliation, on peut notamment penser à la filiation culturelle et communautaire, comme on l'a noté dans les tableaux-fresques de Kent Monkman, par exemple, ou chez les figures d'orpheline et de bâtarde chez les écrivains Jenny Heijun Wills et Éric Dupont.

Certain.es auteur.es n'hésitent pas, comme Leanne Bestamosake Simpson, avec son essai As We Have Always Done, à imaginer une filiation hors de l'humanité, située au côté de la terre et de la nature, à travers ce qu'elle appelle «a land-based pedagogy». D'autres, composent, tel que le fait Jane Willis dans Geniesh: An Indian Girlhood ou Naomi Fontaine, dans Kuessipan, une communauté dans la souffrance; alors que d'autres se disent sœurs et frères dans l'exclusion comme effet des grands paradigmes de la colonisation, dans La Route du lilas d'Éric Dupont. Si les écrivain.es exploré.es conçoivent l'être sous la forme du mouvement, ils se tournent évidemment aussi vers une mise en cause de la fixité et vers l'ouverture à une série de ruptures avec la terre (natale), avec la langue, avec des territoires familiers. Il s'agit donc pour le sujet littéraire contemporain de soutenir le caractère exceptionnel d'un espace fait de mobilités parfois risquées et de filiations intermittentes, plutôt que de favoriser un rapport essentialiste avec un héritage historique ou familial auquel il pourrait se rattacher. Si héritage il y a, il faut le construire continuellement. Cet espace devient alors un espace hospitalier pour toutes/tous celles et ceux qui « ont quitté » leur monde et qui se tournent vers un avenir à inventer.

\section{Références}

ADAMS, Ian. "The lonely death of Chanie Wenjack". MacLeans magazine, February 1, 1967, p. 37-45.

AGASSIZ, Louis, Prof. and Mrs. Journey in Brazil. Boston:Ticknor and Fields, 1869. ANDRADE, Oswald De. Anthropophagies. 1928. Paris : Flammarion, 1982. 
AREA, Lelia. Una biblioteca para leer la Nación. Rosario (Argentina): Beatriz Viterbo editora, 2006.

BERND, Zilá. «Analyse de la vocation transculturelle de la revue Interfaces BrasilCanadá (2001-2014)». Envisager les rencontres transculturelles Brésil-Canada sous la direction de Patrick Imbert et Zilá Bernd. Québec: Presses de l’Université Laval, 2015, p, 37-51.

BERTRAND, Denis, et André LAVALLÉE, dir. Le Rapport Durham. Montréal : Les éditions Sainte-Marie, 1969.

BETASAMOSAKE SIMPSON, Leanne. As We Have Always Done: Indigenous Freedom through Radical Resistance. Minneapolis: University of Minnesota Press, 2017.

BHABHA, Homi. The Location of Culture. London: Routledge, 1993.

BOUCHARD, Gérard. Making of the Nations and Cultures of the New World: an Essay in Comparative History, traduit en anglais par Michelle Weinroth et Paul Leduc. Montréal: McGill-Queen's University Press, 2008.

BOYDEN, Joseph. Wenjack. Toronto: Hamish Hamilton/Penguin, 2016.

BRAIDOTTI, Rosi. « Sur le nomadisme ». Entretien avec Rosi Braidotti. Alternatives Européennes, 2019, https://euroalter.com. Consulté le 29 janvier 2019.

BRAIDOTTI, Rosi. Nomadic Subjects: Embodiment and Sexual Difference in Contemporary Feminist Theory. New York: Columbia University Press, 1994.

Chatelaine. «Two Girlhoods». Rev. of Dobryd by Ann Charney, and Geniesh: An Indian Girlhood, by Jane Willis, October 1973, p. 12.

CHEDA, Sherill. “Geniesh: An Indian Girlhood, Jane Willis, New Press”, The Canadian Forum, August 1974, p. 41-42.

DELEUZE, Gilles, et Félix GUATTARI. Mille Plateaux. Paris : Éditions de Minuit, 1980.

DUCHARME, Réjean. L’Avalée des avalés. Paris : Gallimard, 1966.

DUPONT, Éric. La Logeuse. Montréal: Marchand de feuilles, 2006.

DUPONT, Éric. La Route du lilas. Montréal : Marchand de feuilles, 2018.

FERRON, Jacques. La Nuit. Montréal : Parti pris, 1965.

FONTAINE, Naomi. Kuessipan. Montréal : Mémoire d'encrier, 2017.

GIRARD, René. Des choses cachées depuis la fondation du monde. Paris: Gallimard, 1978.

GOLDSCHLÄGER, Alain, et Clive THOMSON, dir. Le discours scientifique comme porteur de préjugés?/Scientific Discourse as Prejudice-Carrier?. London: The University of Western Ontario-Mestengo Press, 1998. 
IYER, Pico. Imagining Canada, Toronto: Hart House-University of Toronto, 2001.

JOHNSTON, Basil H. Indian School Days. Ontario: Key Porter Books, 1988.

KAPESH, An Antane. Je suis une maudite sauvagesse. Montréal, Leméac, 1976.

KOEVERDEN, Jane Van. "Why Jenny Heijun Wills Wrote a Book about Reuniting with Her First Family in Korea". CBC Books. 18 October 2019. https://www.cbc.ca/ books/why-jenny-heijun-wills-wrote-a-book-about-reuniting-with-her-first-family-inkorea-1.5307845. Browsed on November 20, 2019.

KYMLICKA, Will. Multicultural Odysseys: Navigating the New International politics of Diversity. Oxford: Oxford University Press, 2007.

LAFERRIÈRE, Dany. Cette grenade dans la main du jeune nègre est-elle une arme ou un fruit: Montréal, VLB éditeur, 1993.

MAXWELL, Amélie. "Le mythe américain du self-made man dans les littératures francophones du Canada: apprivoiser le changement en s'approvisionnant de déplacements». Américanité, cultures francophones canadiennes et société des savoirs: Le Canada et les Amériques, sous la direction de Patrick Imbert. Ottawa : Éditions Chaire de l'Université d'Ottawa: “Canada: Enjeux sociaux et culturels dans une société du savoir", 2009, p. 111-142.

MONKMAN, Kent, en collaboration avec Gisèle Gordon. Miss Chief's Memoirs, livret produit pour illustrer l'exposition Kent Monkman, Shame and Prejudice: A Story of Resilience.

MONKMAN, Kent. Site web: https://www.kentmonkman.com. Consulté le 22 novembre 2019.

ORANGE, Tommy. Ici n'est plus ici. Paris: Albin Michel, 2009.

PETRONE, Penny. Native Literature in Canada: From the Oral Tradition to the Present. Toronto: Oxford University Press, 1990, p. 117.

ROBERT, Marthe. Roman des origines et origines du roman. Paris: Gallimard, 1972.

ROBIN, Régine. Cybermigrances. Traversées fugitives. Montréal: VLB éditeur, 2004.

SARMIENTO, Domingo Faustino. Facundo. 1845. Paris: Stock, 1934.

SCHULTZ, Alfred P. Race of Mongrel. Boston: L.C. Page, 1908.

SHAPIRO, Michael J. Violent Cartographies: Mapping Cultures of War. Minneapolis: University of Minnesota Press, 1997.

TAN, Amy. The Joy Luck Club. New York: G. P. Putnam, 1989.

TAYLOR, Charles. Multiculturalism: Examining the Politics of Recognition. Princeton: Princeton University Press, 1994.

THÙY, Kim. Ru. Montréal: Libre Expression, 2009. 
WILLIS, Jane. Geniesh: An Indian Girlhood. Toronto: New Press, 1977.

WILLS, Jenny Heijun. Older Sister. Not Necessarily Related. Toronto: McClelland \& Stewart, 2019.

\section{Notes}

1 Assistant Professor, French, Department of Modern Languages and Literatures, University Winnipeg, Winnipeg, Manitoba, Canada. a.balint@uwinnipeg.ca.

2 Professeur éminent, membre de la Faculté des études supérieures et postdoctorales, Département de Français, Université d’Ottawa, Ontario, Canadá. pimbert@uottawa.ca.

3 The two authors divided the research, writing and review tasks.

4 Voici le résumé du contenu: "A brief history of the rise and fall of the ancient races of earth: a theory that the fall of nations is due to intermarriage with alien stocks: a demonstration that a nation's strength is due to racial purity: a prophecy that America will sink to early decay unless immigration is rigorously restricted”. Nous traduisons: «Une brève histoire de l'ascension et de la chute des races anciennes sur terre : une théorie montrant que la décadence des nations provient des mariages avec des étrangers: une démonstration que la force des nations est due à la pureté raciale: une prévision affirmant que l'Amérique sombrera dans une décadence proche à moins que l'immigration ne soit rigoureusement contrôlée».

5 Nous traduisons: «La résultante naturelle des contacts ininterrompus entre sang mêlés est une classe d'hommes dans lesquels les types purs disparaissent complètement comme le font les belles qualités, physiques et morales, des races primitives, ce qui engendre une foule de bâtards aussi répugnante que des chiens bâtards qui peuvent ainsi être leurs compagnons et parmi lesquels il est impossible de trouver un seul spécimen qui aurait gardé l'intelligence, la noblesse ou l'affection de nature qui rend le chien de race pure le compagnon favori de l'homme civilisé».

6 Pour le racisme et le discours scientifique, voir: GOLDSCHLÄGER ET THOMSON, dir., Le discours scientifique comme porteur de préjugés?/Scientific Discourse as Prejudice-Carrier?.

7 Nous traduisons: «La culture fondatrice ne se maintient pas sur la richesse des autres, mais plutôt sur sa propre pauvreté, sur ses manques réels ou imaginaires, par une subversion calme et vorace».

8 Nous traduisons: «Le Canada doit se considérer comme une nation des bâtards, entièrement constituée et autonome dès sa naissance».

9 Nous traduisons: «Je conclus, enfin, avec une confession : tout au long de ce discours j’ai utilisé un mot hautement déplaisant, 'mongrel', au lieu du mot euphonique et approuvé par le gouvernement, 'hybride' [...] comme le propose Michael Ondaatje, avec son utilisation d'un mot également indifférent, 'bâtards internationaux', le processus de métissage n'est pas toujours plaisant mais il n'est aucunement mauvais si on sort sans pedigree».

10 Nous traduisons: Ma mère croyait qu'on pouvait devenir ce que l'on voulait en Amérique. L'Amérique était l'endroit où tous les espoirs de ma mère se trouvaient. Elle était arrivée ici en 1949 après avoir tout perdu en Chine. Sa mère, son père, sa famille, sa maison, son premier mari, ses deux petites filles, des jumelles. Mais elle n'a jamais regardé en arrière avec regret. Il y avait tellement de manière pour que les choses aillent mieux».

11 Ce n'est qu'en 1988 que le livre Indian School Days de Basil Johnston reçoit une certaine reconnaissance.

12 Nous traduisons: «Le compte rendu de ses premières années et de son éducation par Jane Willis ne s'élève pas au-dessus d'une protestation autobiographique parce qu'elle n'a pas été au-delà de l'amertume et du désir de condamner».

13 C'est de la sorte qu'on échappe aux deux pôles qui peuvent contribuer à maintenir les Autochtones dans une certaine forme de marginalisation, comme le souligne le narrateur dans Ici n'est plus ici de Tommy Orange où il parle d'un groupe de DJ canadiens issus des Premières Nations et installés à Ottawa: «L'obstacle, ou l'impasse dans tout ça, le voilà: si ça ne s'inspire pas de la tradition, en quoi est-ce autochtone? Et si c'est trop fidèle à la tradition, au passé, en quoi est-ce pertinent pour les peuples autochtones d'aujourd'hui, en quoi est-ce moderne?» (p. 93).

14 Voir le site web de Kent Monkman : https://www.kentmonkman.com.

15 Nous traduisons: «Ma mission est de faire reconnaître l'expérience autochtone dans le canon de l'histoire de l'art qui nous a ainsi perdu de vue». 
16 Nous traduisons: «Cela m'a conduit à réfléchir à ce qu'est l'hétérosexualité dans des sociétés où être queer est normalisé, où la différence n'est pas perçue comme différence, mais comme norme».

17 Nous traduisons: «Le sujet nomade est un mythe, c'est-à-dire une fiction politique qui me permet de penser et de me déplacer à travers des catégories établies et différents niveaux d'expérience: en transgressant des frontières sans brûler des ponts».

18 Nous traduisons: «Le choix d'une figure iconoclaste et mythique comme le sujet nomade s'avère être une remise en question de la théorie dominante et particulièrement, de la pensée philosophique. Cette figure traduit donc mon désir d'explorer et de légitimer l'agentivité politique en prenant comme preuve historique le déclin des identités ontologiquement fixes et solides. Une des questions de base ici est de réconcilier le fragmentaire et la discontinuité avec l'invention de nouvelles formes d'interconnectivité et de projets politiques communs».

19 Nous traduisons: «Le nomade ne représente pas le sans-abri ou le déracinement compulsif; il est plutôt la figuration d'un sujet qui a abandonné toute idée, désir et nostalgie pour la fixité».

20 Nous traduisons: «Le sujet nomade [...] n'est pas dépourvu d'unité; son mode de fonctionnement est celui des paradigmes précis et temporaires de la mobilité, plutôt que celui des routes fixées d'avance. Il s'agit d'une cohésion issue de la répétition, des mouvements cycliques, des déracinements rythmés».

21 Betasamosake Simpson écrit qu'elle a recours aux "Nishnaabeg stories to advocate for a reclamation of land as pedagogy, both as process and context for Nishnaabeg intelligence, in order to nurture a generation of Indigenous peoples that have the skills, knowledge and values to rebuild our nation according to the world views and values of Nishnaabeg culture" (As We Have Always Done, p. 35). Nous traduisons: «histoires Nishnaabeg pour défendre une pédagogie basée sur la réclamation de la terre, à la fois comme processus et comme contexte pour l'intelligence Nishnaabeg, afin de nourrir une génération de peuples autochtones qui ont les capacités, les savoirs et les valeurs pour reconstruire notre nation selon les visions du monde et les valeurs de la culture Nishnaabeg».

22 Nous traduisons: «Comme style intellectuel, le nomadisme ne consiste pas autant dans le fait de ne pas avoir d'habitation que dans la capacité de se créer une habitation partout. Le nomade transporte ses affaires de base partout où il ou elle va et peut se créer une habitation de fortune n'importe où». 